\title{
Mitochondrial functionality and beef colour: A review of recent research
}

\author{
R. Ramanathan ${ }^{1 \#}$, M.N. Nair ${ }^{2}$, M.C. Hunt ${ }^{3}$ \& S.P. Suman ${ }^{4}$ \\ ${ }^{1}$ Department of Animal and Food Sciences, Oklahoma State University, Stillwater, OK 74078, USA \\ ${ }^{2}$ Department of Animal Sciences, Colorado State University, Fort Collins, CO 80523, USA \\ ${ }^{3}$ Department of Animal Sciences and Industry, Kansas State University, Manhattan, KS 66506, USA \\ ${ }^{4}$ Department of Animal and Food Sciences, University of Kentucky, Lexington, KY 40546, USA
}

(Received 5 July 2018; Accepted 13 November 2018; First published online 2 March 2019)

\begin{abstract}
Copyright resides with the authors in terms of the Creative Commons Attribution 4.0 South African Licence.
See: http://creativecommons.org/licenses/by/4.0/za

Condition of use: The user may copy, distribute, transmit and adapt the work, but must recognise the authors and the South African Journal of Animal Science.
\end{abstract}

\begin{abstract}
The bright-red colour of meat that consumers prefer depends on the depth of oxygen diffusion into the tissue and myoglobin oxygenation. Interestingly, both processes are influenced by mitochondrial activity in postmortem muscle. The transition of muscle metabolism from aerobic to anaerobic pathways affects various cellular processes including mitochondrial functionality. Numerous studies report that even with compromised structure, mitochondria continue to influence postmortem beef colour via oxygen consumption and metmyoglobin reducing activity. Hence, an in-depth understanding of the pre- and post-harvest factors affecting mitochondrial functionality can significantly enhance existing knowledge of meat colour and colour stability. Several comprehensive reviews have discussed the biochemical factors affecting meat colour, but there are only a few that have sections on the impact of mitochondria on beef colour. Furthermore, advancement of high-throughput techniques such as metabolomics and proteomics has enabled researchers to elucidate metabolite and protein changes related to mitochondria. Therefore, the objective of this review is to provide an overview on the role of mitochondria in beef colour, with a focus on recent advances in mitochondrial research, oxygen consumption, and metmyoglobin reducing ability.
\end{abstract}

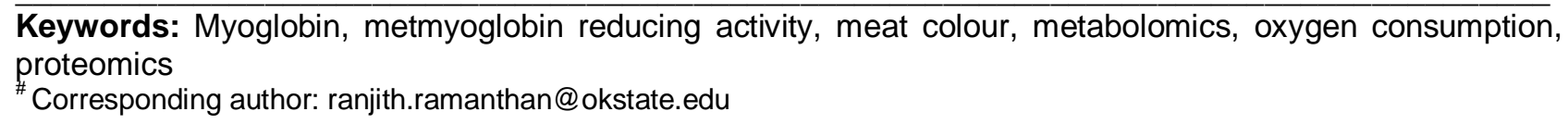

\section{Introduction}

A bright cherry-red colour is a highly desirable beef quality trait during grading, fabrication, value addition and retail sales. Consumers often use colour as an indicator to gauge the freshness and wholesomeness of beef, and any deviation from a bright-red colour often leads to discounted value of meat (Faustman \& Cassens, 1990; Mancini \& Hunt, 2005; Suman et al., 2014).

Myoglobin is the primary sarcoplasmic protein that determines meat colour along with hemoglobin and cytochrome $c$, although the latter two to a lesser extent. Depending on the heme redox state and the ligand attached, myoglobin can exist in three forms, namely deoxy-, oxy- and metmyoglobin (AMSA, 2012). When deoxymyoglobin predominates, the meat will be a darker, purple colour not commonly accepted by consumers, while oxymyoglobin gives the bright-red colour preferred by consumers. Metmyoglobin is responsible for browning and discolouration of meat at nearly every stage of the meat delivery chain.

Mitochondria are essential organelles involved in energy production in live animals. In postmortem muscles, mitochondria remain functional and can influence myoglobin redox chemistry (Tang et al., 2005a; Mancini \& Ramanathan, 2014). Formation of a bright-red colour depends on oxygen diffusion into the meat surface and oxygen consumption by the muscle, both of which in turn are influenced by mitochondrial functionality (Ramanathan et al., 2010a). Hence, understanding the factors that influence mitochondrial function is critical to minimize the economic losses related to the appearance of beef.

Several excellent reviews have discussed myoglobin chemistry, factors affecting discolouration, metmyoglobin reducing activity and the role of mitochondria in beef colour (Giddings, 1977; Cornforth, 1994; 
Bekhit \& Faustman, 2005; Mancini \& Hunt, 2005; Suman \& Joseph, 2013). This review will provide an overview of the recent advances in mitochondrial research related to beef colour and its stability.

\section{Biochemistry of mitochondrial oxygen consumption and beef colour}

Mitochondria and myoglobin chemistry are highly interrelated. Oxygen consumption (OC) and metmyoglobin reducing activity (MRA) are two critical biochemical processes that influence beef colour (Ledward, 1985; Sammel et al., 2002a; Seyfert et al., 2007; English et al., 2016a). Although MRA and OC impact myoglobin redox states differently, both processes are interconnected and are influenced by mitochondrial activity (Tang et al., 2005a; Ramanathan et al., 2013; Ke et al., 2017).

\section{Oxygen consumption}

Physiologically, myoglobin serves as the oxygen reservoir in muscle that can be used by the mitochondria for energy production/oxidative phosphorylation (Wittenberg \& Wittenberg, 1989). When needed, oxymyoglobin transfers its oxygen to mitochondria. In postmortem muscle, heme proteins, mitochondria, oxygen-consuming enzymes (OCE), microbes, and lipid oxidation compete for available oxygen (Faustman \& Cassens, 1991; Baron \& Anderson, 2002; Tang et al., 2005). The role of microbes and lipid oxidation in oxygen consumption is negligible with good hygiene (DeVore, 1974). However, the competition between myoglobin oxygenation and mitochondria/OCE for available oxygen influences beef colour (Tang et al., 2005; Ramanathan et al., 2013). In meat science, the term "OC" refers to oxygen consumed primarily by mitochondria and OCE. A similar terminology, oxygen consumption rate (OCR), also appears in literature. Researchers should note that the $O C$ indicates absolute differences in $O C$ at a set time point, whereas OCR expresses OC per unit of time. The latter protocol is more tedious, especially if large numbers of samples are involved.

Mitochondrial oxygen consumption occurs at the terminal stage of the electron transport chain. Utilization of co-factors such as NADH and substrates such as citrate, glutamate, malate, pyruvate, and succinate can increase mitochondrial OC (Atkinson, 1969; Tang et al., 2005; Mohan et al., 2010a; Bjelanovic et al., 2016). To maximize bloom as a bright-red colour, a moderate OC is needed because if OC is too high the bloom decreases, and if $\mathrm{OC}$ is too low the metmyoglobin content increases which reduces the reservoir of ferrous heme pigment that can be oxygenated (Sammel et al., 2002b; Mancini \& Hunt, 2005). Pre-rigor meat fails to fully bloom due to its higher OC, compared with post-rigor meat.

The role of mitochondria in bloom development has been validated by the addition of mitochondrial inhibitors such as rotenone to meat homogenates in in vitro systems (Cornforth \& Egbert, 1985). Rotenone treatment of pre-rigor muscle homogenates resulted in an approximately $75 \%$ increase in redness compared with control pre-rigor meat homogenates. Another example of failure to fully bloom is lactate-enhanced beef. Although both physical and biochemical factors can lead to muscle darkening with lactate-enhancement, the research assessing the mechanism(s) of the darkening indicated that the role of mitochondria was due to the lowering of myoglobin oxygenation (Ramanathan et al., 2010a). More specifically, the addition of lactate can regenerate NADH via lactate dehydrogenase activity (Kim et al., 2006). NADH can promote mitochondrial oxygen consumption resulting in darkened muscle (lower $L^{*}$ values). The role of mitochondria in lactateinduced darkening was also demonstrated by the addition of rotenone (mitochondrial complex I inhibitor) to muscle homogenates (Ramanathan et al., 2009). The addition of rotenone minimized potassium lactateinduced muscle darkening by $13 \%$.

In vitro research further confirmed that functional mitochondria can promote deoxymyoglobin formation and muscle darkening. For example, the addition of succinate to the mitochondria-oxymyoglobin mixture at $\mathrm{pH} 5.6$ promoted deoxymyoglobin formation (Tang et al., 2005). Succinate is a complex II substrate of the electron transport chain, which can enhance mitochondrial activity. Conversely, the addition of antimycin A (electron transport chain complex III inhibitor) minimized oxygen consumption and deoxymyoglobin formation. The efficient conversion of oxy to deoxymyoglobin depends on a greater $\mathrm{pH}$, elevated temperature, and/or a greater concentration of mitochondria, plus a functional metmyoglobin reducing system.

Previous research reported that a higher postmortem muscle $\mathrm{pH}$ is the single most important factor that can prolong mitochondrial functionality (Giddings, 1977). Furthermore, the mitochondria from darkcutting beef had progressively greater oxygen consumption as the $\mathrm{pH}$ of dark-cutting meat increases, and the extent of the bloom decreases due to less pigment oxygenation (Ashmore et al., 1972; English et al., 2016b). Although the depletion of glycogen in animals prior to slaughter is the main reason for the limited decline of post-rigor muscle $\mathrm{pH}$, McKeith et al. (2016) reported that severe dark-cutting beef had a greater mitochondrial content and that the mitochondria were less efficient in electron transport as indicated by 
greater reactive oxygen species formation than normal $\mathrm{pH}$ beef mitochondria. These authors suggest that less efficient mitochondria lead to the more rapid depletion of glycogen during ante-mortem stress.

Wet-aging is a common practice to improve beef tenderness due to proteolysis and weakening of the structural integrity of sarcomeres. Aging is affected by numerous factors such as muscle aging time and temperature, muscle $\mathrm{pH}$, packaging (especially the atmosphere), animal age, nutritional regimen, animal genetics, microbial issues, and the metabolic state of the muscles resulting from an interaction of the rate of $\mathrm{pH}$ decline and carcass chilling during the onset of rigor. Several of these factors also affect meat colour.

Numerous studies reported details about beef colour and/or colour stability during postmortem aging (O'Keeffe \& Hood, 1982; Ledward, 1985; Madhavi \& Carpenter, 1993; Cornforth, 1994; McKenna et al., 2005; Seyfert et al., 2007; King et al., 2012; Mancini \& Ramanathan, 2014; English et al., 2016a; Neethling et al., 2016). In general, there is agreement that meat aged and displayed early postmortem $(\approx 2$ weeks) will bloom less but have a greater colour life than meat that is aged $>3$ to 6 weeks. Conversely, extended aging of beef will have greater bloom but a shorter colour life. This observation varies considerable from muscle to muscle and several of the factors mentioned above. There is also general agreement that muscles can be categorized as having high, medium, or poor colour stability of intact muscle (McKenna et al., 2005) and for ground beef containing mixtures differing in colour-stable muscles (Raines et al., 2010).

Theoretically, in early postmortem the mitochondria should be more active, thus less extensive blooming and greater colour life due to more active MRA. However, in later postmortem the OC would decrease resulting in greater oxymyoglobin formation but the colour stability declines due to less functional MRA colour chemistry. Recent work by English et al. (2016a) clearly supports earlier observations about early and late combinations of factors. They found that extended aging of beef longissimus muscle (> 21 days) failed to bloom compared with steaks aged for 7 or 14 days. Redness in loins aged for 62 days decreased by $26 \%$ and $7 \%$ compared with 21 -day aged loins when packaged and displayed in PVC and HiOx-MAP (high-oxygen modified atmospheric packaging; $80 \%$ oxygen and 20\% carbon dioxide), respectively (English et al., 2016a). Aging appears to increase mitochondrial damage and depletes substrates for OC and MRA (Tang et al., 2005). This suggests that, in part, a major role for mitochondria during bloom development where a greater MRA and "intermediate" OC are critical for an extended brightred colour life. Furthermore, lower bloom in extended aged beef can be attributed to partial myoglobin denaturation leading to lower myoglobin concentration. Myoglobin denaturation can increase the sarcoplasmic concentration of free ferrous or ferric non-heme iron, which can decrease mitochondrial activity. Recently, Purohit et al. (2014) reported that the addition of inorganic ferrous and ferric iron decreased mitochondrial utilization of succinate and lactate and the mitochondrial OC.

Utilization of case-ready meats to lower labour costs and extend product colour and shelf life has capitalized on the functional benefits of modified atmosphere packaging (MAP). These packaging systems range from conventional vacuum packaging (purple colour) with no head space to those with head spaces containing blends of oxygen (bright-red colour and high oxygen), nitrogen (for filler), carbon dioxide (for antimicrobial), and carbon monoxide (bright-red colour and very low oxygen). The gas blends typically used are either high oxygen or very low oxygen (essentially none). The success of these systems is critically dependent upon meat's ability to consume oxygen and to reduce metmyoglobin.

Technically, the high-oxygen system compared to low-oxygen systems is the simplest to utilize because the meat is exposed to 3 to 4 times more oxygen than that in air, thus excellent bloom is obtained by increasing the thickness of the oxymyoglobin on the surface of the meat. Numerous studies report that high-oxygen packaging increases colour life by 2 to 4 times compared to atmospheric packaging (Jayasingh et al., 2002; Seyfert et al., 2007).

To manage low-oxygen systems a solid working knowledge of the interconversions of the myoglobin redox pigments is needed (Section II, AMSA, 2012). When a steak with an oxygenated surface is packaged in a vacuum or in anaerobic packaging such as CO-MAP, mitochondria must first consume oxygen to lower the oxygen partial pressure in the package, resulting in deoxymyoglobin. Carbon monoxide binds with deoxymyoglobin as it cannot bind with oxymyoglobin or metmyoglobin. This may explain why CO-MAP steaks require 48 to $72 \mathrm{~h}$ to present with the characteristic bright-red colour (Jayasingh et al., 2001). Thus, for effective OC, i.e., the conversion of oxymyoglobin to deoxymyoglobin, MRA is also a critically important biochemical process that must occur simultaneously to establish conditions for the reduction of metmyoglobin leading to the ultimate formation of redox forms. More specifically, myoglobin is particularly prone to oxidation around 1 and $6 \mathrm{~mm} \mathrm{Hg}$ partial pressure in purified form and in intact steak, respectively (George \& Stratmann, 1952; Ledward, 1970). Formation of metmyoglobin is evident in vacuum-packaged steaks or extended-aged steaks packaged in a vacuum. During OC, oxygen partial pressure drops to this range and myoglobin is particularly prone to oxidation. However, if meat has enough MRA and if the meat temperature is sufficiently warm for the chemistry to run, this reaction may not be visible in fresh meat, hence 
visible accumulation of metmyoglobin will be less and the desired redox forms will be greater (see Section II, AMSA, 2012 for details).

\section{Metmyoglobin reducing activity}

Metmyoglobin reducing activity refers to the ability of the muscle to convert metmyoglobin to deoxymyoglobin under two conditions: 1) the transfer of an electron by enzymatic, non-enzymatic, or electron-transport mediated pathways (Arihara et al., 1995; Tang et al., 2005; Elroy et al., 2015); and 2) the lowering of oxygen via muscle OC chemistry. Metmyoglobin reducing activity is critical for effective oxygen transport in live animals. During transfer of oxygen from oxymyoglobin to the mitochondria, some of the myoglobin could get oxidized to metmyoglobin at the outer mitochondrial membrane. Metmyoglobin is incapable of carrying oxygen for oxidative phosphorylation. However, the inherent capacity of the muscle tissue helps convert metmyoglobin to deoxymyoglobin. These pathways remain active in postmortem muscles, but the time and efficiency of MRA vary considerably among muscles (Ledward, 1985; Madhavi \& Carpenter, 1993; McKenna et al., 2005)

In enzymatic metmyoglobin reduction, NADH-dependent cytochrome b5 reductase transfers an electron from NADH via electron carrier for metmyoglobin reduction (Djimsa et al., 2017). Arihara et al. (1995) demonstrated localization of NADH-dependent cytochrome b5 reductase within the mitochondrial outer membrane. Moreover, the mitochondrial fraction from the gluteus medius had greater NADHdependent cytochrome b5 reductase activity than the longissimus dorsi muscle indicating muscle-specificity in MRA (Lanari \& Cassens, 1991).

In non-enzymatic metmyoglobin reduction, artificial electron carriers such as methylene blue or cytochrome b5 can transport electrons. In mitochondria-mediated MRA, electrons available between complex III and IV are transferred to metmyoglobin by cytochrome $c$ and outer membrane cytochrome $b 5$ (Tang et al., 2005; Ramanathan et al., 2010c). Furthermore, mitochondria have the ability to regenerate NADH with the help of complex I, NAD, and succinate (Beleski et al., 2015b). This reaction occurs when complex III is not functional. Klingenberg (1968) noted that sub-mitochondrial particles could donate electrons from succinate or cytochrome $c$ to NAD. Mitochondrial outer membrane contains a NADHdependent reductase enzyme; hence, the presence of NADH can result in enzymatic metmyoglobin reduction. Clearly, NADH and a variety of substrates can act as reducing agents that can directly influence myoglobin redox changes. For example, NADH can donate an electron and this can be utilized for enzymatic, non-enzymatic, and mitochondria-mediated metmyoglobin reduction. Similarly, several tricarboxylic acid metabolites can be used for electron-transport mediated metmyoglobin reduction.

Metmyoglobin reducing activity can occur by aerobic and anaerobic processes. Ledward (1973) reported that aerobic metmyoglobin reducing activity has a greater correlation with metmyoglobin formation during display $(r=-0.94)$ than anaerobic $(r=-0.22)$ metmyoglobin reducing activity. Similar results have also been reported in in vitro studies using metmyoglobin, mitochondria, and succinate. Although the mechanism is not clear, it appears that to finalize the reduction of metmyoglobin requires both OC and MRA to work cooperatively. Furthermore, electrons available between complex III and IV with the utilization of succinate and lactate can be donated to metmyoglobin via cytochromes $c$ and $b 5$ leading to effective metmyoglobin reduction. OC is more sensitive than MRA. Ledward (1972) concurs that oxygen partial pressure is more decisive in discolouration than MRA.

Previous research hypothesized that the addition of glycolytic and tricarboxylic acid metabolites could generate reducing equivalents such as NADH for metmyoglobin reduction. Lactate enhancement in caseready meat was a practical application of this concept (Mancini et al., 2004; Kim et al., 2006). Enhancing beef with lactate improved beef colour primarily by regeneration of NADH via lactic dehydrogenase activity. The NADH formed can be utilized for enzymatic, non-enzymatic, and mitochondria-mediated pathways (Ramanathan et al., 2010d). Several other studies have also demonstrated that the addition of substrates such as glutamate and malate can enhance MRA and meat colour. More specifically, malate dehydrogenase can convert malate to oxaloacetate with the regeneration of NADH (Mohan et al., 2010b). However, it is critical to understand the biochemical metabolism of each substrate. For example, the action of lactic dehydrogenase is reversible depending on the product concentration. Enhancing beef with pyruvate discoloured steaks when packaged in a vacuum, while the colour improved when packaged in HiOx and PVC (Ramanathan et al., 2011). Conversion of lactate to pyruvate is a reversible reaction with the regeneration of $\mathrm{NADH}$ via lactic dehydrogenase. However, this reaction is dependent on the concentration of either lactate or pyruvate. If the concentration of pyruvate in tissue is greater, the NADH content can be depleted because pyruvate is converted to lactate with the use of NADH. Similarly, the addition of pyruvate to muscle homogenate had the least effect on redness compared with malate and lactate (Mohan et al., 2010a). Packaging-specific effects of malate, glutamate, succinate, pyruvate, and citrate on ground beef colour was also reported by Bjelanovic et al. (2016). These authors noted that a mixture of succinate and 
glutamate in anaerobic packaging was very effective in deoxymyoglobin formation, while glutamate, malate, and citrate were effective in maintaining a red colour in aerobic packaging. However, pyruvate discoloured patties when packaged in vacuum.

Aging had a packaging-specific effect on colour stability (English et al., 2016a). When loins were aged for 62 days, HiOx-MAP had a lower redness than PVC by the end of a six-day retail display. Interestingly, loins aged for 62 days and steaks packaged in CO-MAP had lower redness at the end of a six-day retail display than 21-day aged steaks packaged in CO-MAP (7.6\% reduction in $\mathrm{a}^{*}$ values). The decrease in colour stability can be attributed to depletion of metabolites required for regenerating NADH, mitochondrial damage/activity (Tang et al., 2005), decreased MRA (Mancini \& Ramanathan, 2013), and reduced antioxidant status of muscles.

\section{Muscle-specific effects on mitochondrial function}

Meat discolouration is a muscle-specific attribute for intact muscle (Lawrie, 1961; McKenna et al., 2005) and for ground product (Raines et al., 2010); more specifically, the proportions of red and white fibres which can influence colour stability. Psoas major (PM: predominant red fibres) has a shorter colour stability than longissimus lumborum (LL: more white fibres) during retail display in PVC and HiOx packaging. Predominant red fibres will have oxidative metabolism with more myoglobin and mitochondria (Hunt \& Hedrick, 1977). Mitochondrial content is approximately 1.2 - 2.5-fold greater in PM than in LL (Mohan et al., 2010; Ke et al., 2017). Although various oxidative processes can be attributed to shorter colour stability in $\mathrm{PM}$, a recent study suggests that there are muscle-specific differences in psoas and longissimus mitochondrial properties. For example, oxygen consumption and MRA of bovine PM mitochondria were greater during initial display time than LL when packaged in PVC (Belskie et al., 2015a). However, both parameters decreased rapidly in PM compared with LL during a seven-day display. The muscle-specific effect on OC and MRA can be attributed to mitochondrial degeneration rates during storage. For example, Ke et al. (2017) reported that PM mitochondrial content decreased $68 \%$ compared with $28 \%$ in LL by the end of a seven-day simulated retail display. The cytochrome $c$ content in the sarcoplasm increased more in PM than in LL (Ke et al., 2017). Cytochrome $c$ is the major electron carrier between complex III and IV of the electron transport chain and used as an indicator of mitochondrial degeneration. This suggests that musclespecific factors can influence mitochondrial degeneration and beef colour. However, knowledge to date is limited to the factors affecting mitochondrial degeneration in postmortem muscles.

Beef semimembranosus is a large muscle in beef hindquarter which exhibits intramuscular variations in colour stability and can be separated into a colour-stable outside (OSM) and a colour-labile inside (ISM; Sammel et al., 2002a). The variations in temperature and pH decline during carcass chilling are considered reasons for the variation in colour stability and muscle chemical functionality (Sammel et al., 2002a; b). Moreover, the differential abundance of glycolytic enzymes also contributes to the differential $\mathrm{pH}$ fall rate between ISM and OSM (Nair et al., 2016). Recent studies by Nair et al. (2017) reported variation in functionality of mitochondria isolated from OSM and ISM. OSM had a greater mitochondrial OC, and it corresponded with its greater colour stability, possibly due to its ability to reduce metmyoglobin to ferrous forms for longer periods.

\section{Quantification of oxygen consumption and metmyoglobin reducing ability}

Despite being a post-rigor entity, meat remains biochemically active; hence, accurate measurements of oxygen consumption and MRA will be challenging. The problem is to solve the dynamics of the cellular organelles and the chemistry that are now operating at a lower $\mathrm{pH}$, considerably more uniform concentration gradients, more permeable membranes, and substrate concentrations that seem to diminish as postmortem age increases, and with myriad processing protocols. However, quantification of oxygen utilization and metmyoglobin reduction in meat is essential to understand the role of mitochondria in meat colour. Oxygen consumption studies utilizing isolated mitochondria or using intact steaks can provide valuable information as to how various pre- and postharvest processes can influence mitochondrial function. Different types of tests performed using mitochondrial fraction are summarized in Table 1. Each method has its own advantages and disadvantages. Hence, method selection depends on the objectives of the study.

\section{In vitro mitochondrial oxygen consumption}

Mitochondria are membrane-bound organelles involved in oxidative phosphorylation. Immediately after harvest, metabolism changes from aerobic to anaerobic which affects muscle homeostasis. Interestingly, mitochondria can consume oxygen even with compromised function/morphology. Various in vitro studies have shown that isolated mitochondria can consume oxygen with the addition of mitochondrial complexspecific substrates such as pyruvate-malate or pyruvate-glutamate NADH, (complex I), succinate (complex II), pyruvate, ascorbate/TMPD (complex IV), malate, and glutamate. Furthermore, the individual roles of 
electron-transport chain complexes can be studied with the help of specific inhibitors such as rotenone (complex I), malonate (complex II), antimycin A (complex III), and azide/cyanide (complex IV). The stability of different complexes postmortem determines substrate utilization. Giddings (1977) pointed out that complex II is more labile than other complexes with storage. Hence, succinate may be utilized at a lower rate.

Table 1 Different types of biochemical assays performed to study mitochondrial function

\begin{tabular}{|c|c|c|c|}
\hline & & Purpose & Reference \\
\hline 1 & Isolated mitochondria & Mitochondrial activity/oxygen consumption & Ashmore et al., 1972 \\
\hline 2 & Intact steak & Mitochondrial activity/oxygen consumption & Seyfert et al., 2007 \\
\hline 3 & Mitochondria and myoglobin & In vitro study showing conversion of $\mathrm{OMB}$ to $\mathrm{DMB}$ & Tang et al., 2005 \\
\hline 4 & Cytochrome $c$ & Mitochondrial degeneration & Ke et al., 2017 \\
\hline 5 & Electron microscopy & Structural changes & Cheah \& Cheah, 1971 \\
\hline 6 & Oxygen penetration & Mitochondrial activity/oxygen consumption & O'Keeffe \& Hood, 1982 \\
\hline 7 & Mitochondrial protein content & Mitochondrial degeneration/ protein content & Mohan et al., 2010 \\
\hline 8 & Citrate synthase & Mitochondrial activity & Lanari \& Cassens, 1991 \\
\hline 9 & Cytochrome $c$ oxidase & Mitochondrial activity & Mohan et al., 2010b \\
\hline 10 & Mitochondria-dependent reductase & Metmyoglobin reducing activity & Lanari \& Cassens, 1991 \\
\hline 11 & DNA-based methods & Mitochondrial quantification & Ke et al., 2017 \\
\hline
\end{tabular}

Differential centrifugation is a conventional method to isolate mitochondria from different muscles (Smith, 1967). Although this method can provide valuable information about the role of isolated mitochondria; various steps involved in isolation such as grinding, centrifugation, and protease can affect the structural and chemical integrity of mitochondria. Furthermore, mitochondrial properties are studied in vitro and substrates are added externally. Hence, this may not represent in situ conditions. Clark oxygen electrodes are routinely used instruments to measure oxygen consumption. This method involves the addition of buffer, mitochondria, and substrate in a closed chamber. Consumption of oxygen from added solvents may bias results as an extra electric current is generated, which is proportional to oxygen consumed by mitochondria (Estabrook, 1967). Recently, high-resolution oxygen sensors with automatic sample addition have significantly enhanced the sensitivity and accuracy of oxygen consumption measurements (Phung et al., 2013).

Electron microscopic studies are used to visualize structural changes during storage (Cheah \& Cheah, 1971; Tang et al., 2005; Ramanathan et al., 2012). Various morphological changes such as vaculation and swelling were noted in beef heart mitochondria during 45-d storage. Furthermore, electron microscopic studies help understand the effects of lipid oxidation products such as 4-hydroxy-2-noneal on mitochondrial function and structure. Mitochondria are the first organelle affected by oxidative stress and free radical formation. Hence, in cooperating structural studies with functional studies can help understand the physiology of mitochondria in beef colour.

\section{In situ muscle oxygen consumption}

Intact muscle oxygen consumption offers real-time monitoring of muscle tissue oxygen consumption. Various techniques have been used to quantify $\mathrm{OC}$ in intact steaks (Table 2). Ease, repeatability, $\mathrm{pH}$, and the type of fibre in muscle should be considered when selecting a method for $\mathrm{OC}$. A greater $\mathrm{pH}$ results in swollen muscle fibres; hence, less oxygen penetrates deep into the myofibre, which can influence oxymyoglobin formation. Furthermore, a greater $\mathrm{pH}$ can promote mitochondrial oxygen consumption. In general, type I muscle fibres will have a smaller diameter, which can influence oxygen penetration. These factors should therefore be considered when selecting a method for oxygen consumption. 
Table 2 Different techniques used to quantify oxygen consumption in muscle tissue

\begin{tabular}{llll}
\hline Type of study & Basis of technique & Name of instrument used & Reference \\
\hline \multirow{2}{*}{ Isolated mitochondria } & Differential centrifugation & Clark electrode & Tang et al., 2005 \\
& Permeabilized tissue & High-resolution respirometer & Phung et al., 2011 \\
& Differential centrifugation & High-resolution respirometer & Phung et al., 2011 \\
\cline { 2 - 4 } Intact/minced muscle & Changes in OMB level & Near-infrared technology & Mohan et al., 2010cd \\
& Changes in carbon dioxide & Gas analyzer & McKenna et al., 2004 \\
& Changes in DMB level & Visible spectrophotometer & English et al., 2016 \\
& Changes in OMB level & Visible spectrophotometer & Madhavi et al., 1993 \\
& Changes in oxygen level & Warburg monomeric technique & Bendall 1972 \\
& Changes in oxygen level & Gas analyzer & Sammel et al., 2002b \\
& Resistance to form OMB & Visible spectrophotometer & Ramanathan et al., 2012 \\
& Depth of oxygen penetration & Caliper & Joseph et al., 2012 \\
\hline
\end{tabular}

Both absorbance and reflectance values can be used to quantify oxygen consumption. Changes in the oxymyoglobin level following the vacuum packaging of fresh meat can indirectly indicate oxygen consumption by mitochondria. However, for meat with a higher-than-normal $\mathrm{pH}$, changes in oxymyoglobin formation may not provide accurate information about oxygen consumption. Hence, some researchers have utilized the resistance to form oxymyoglobin as an indicator of oxygen consumption (McKenna et al., 2005; English et al., 2016; McKeith et al., 2016). Mohan et al. (2012d) utilized NIR-based techniques to quantify oxymyoglobin content, which is a critical factor in muscle fibre orientation.

Use of headspace analyzers to quantify gas compositions within a package can also provide valuable information about muscle respiration. If the number of samples for analysis is large, use of a headspace analyzer may limit its application. Spectrophotometric methods and head-space analysis techniques can be related to surface oxygen consumption. However, it will be challenging to quantify the oxygen consumption ability of an entire sample of tissue. Measurement of oxygen penetration into tissue was also used as a tool to indicate oxygen consumption. For example, a greater oxygen consumption in the tissue can limit the depth of oxygen penetration (O'Keeffe \& Hood, 1982). Recently, oxygen sensors such as fibre optic probes connected with oxygen sensors are also used to quantify oxygen levels. Use of oxygen sensors coupled with probe type spectrophotometers will provide a better understanding of oxygen utilization in meat. Tissue oximetry (Mohan et al., 2010d) could provide interesting insight into the chemistry occurring just below the meat surface.

\section{Application of proteomics and metabolomics in mitochondrial research}

Mitochondrial function can be influenced by metabolite concentration and the activity of enzymes involved in TCA cycle and glycolytic pathways (Tang et al., 2005; Ramanathan et al., 2013). Traditional wetlaboratory techniques which characterize the array of metabolites/proteins at a single point in time are challenging and time-consuming. Use of high-throughput techniques such as proteomics and metabolomics will help unravel metabolite/protein changes in muscles during the postmortem period (Joseph et al., 2012; Abraham et al., 2017). Equally critical to this research approach is to collect accurate and precise measurements of meat colour and other phenotypic meat traits that the "omics" will be compared to.

\section{Application of metabolomics in mitochondrial research}

Metabolomic techniques allow simultaneous quantification of hundreds of metabolites such as amino acids, TCA, and glycolytic intermediates in a single run. Various platforms such as gas chromatography, liquid chromatography or nuclear magnetic resonance coupled with mass spectrometry are used to separate metabolites. A recent study utilized metabolomics techniques to determine the role of differentially abundant metabolites in colour stability in LL and PM (Abraham et al., 2017). The results suggest that fructose, glucose 6-phosphate, and pyruvic acid were more abundant in LL than in PM. Furthermore, citric acid was greater in LL than in PM on d 7. Interestingly, the differential abundance in metabolites can increase mitochondrial activity and MRA. Furthermore, the malonic acid level in the psoas major muscle was greater 
than in the longissimus muscle on days 3 and 7 . Malonic acid is a complex II inhibitor and can oxidize $\mathrm{NADH}$, which in turn can negatively affect meat colour.

In another study, Subbaraj et al. (2016) utilized a hydrophilic interaction liquid chromatography-mass spectrometry (HILIC-MS) to study colour stability in ovine meat. They concluded that NADH, malic acid, and guanosine levels were significantly greater in colour-stable ovine muscles than in colour-labile muscles. High-performance liquid chromatography-electron spray ionization-mass spectrometry to differentiate non-polar metabolites of aged beef indicated a decrease in NADH and glutathione concentration in beef muscles with increasing aging time (Ma et al., 2017); thus, monitoring the changes in metabolites that can provide valuable insights into mitochondrial activity and beef colour.

\section{Application of proteomics in mitochondrial research}

Recent research by Mancini et al. (2016) examined the mitochondrial proteome profiles of two muscles differing in colour-stable muscles. The LL is colour stable whereas the PM is a colour-labile muscle. The results indicated that the mitochondrial protein abundance varied between these muscles on day 3 of storage. Among the eight proteins identified as differentially abundant, succinyl-CoA ligase, ubiquinone biosynthesis protein COQ9, ATP synthase, ES1 protein homolog, and very long-chain specific acyl-CoA dehydrogenase were over-abundant in the PM, whereas carbonic anhydrase, creatine kinase S-type, and aspartate aminotransferase were over-abundant in the LL (Mancini et al., 2016). The question remains as to whether these associations are meaningful.

Other studies have reported the differential abundance of sarcoplasmic proteins in relation to meat colour (Joseph et al., 2012; Canto et al., 2015; Nair et al., 2017). Among the proteins identified as differentially abundant between colour-stable and colour-labile muscles, there were several enzymes associated with energy metabolism and glycolytic pathway. These enzymes play a critical role in maintaining mitochondrial functionality in postmortem muscles. Moreover, several enzymes that were specific to mitochondria were detected in the sarcoplasmic fraction, indicating variation in mitochondrial degradation between the muscles (Joseph et al., 2012; Nair et al., 2017a). For example, Joseph et al. (2012) reported a greater abundance of mitochondrial aconitase in the sarcoplasmic proteome of PM compared with LL, and it exhibited a negative correlation with redness. Furthermore, Nair et al. (2017b) reported that several mitochondrial proteins (mitochondrial malate dehydrogenase, mitochondrial ATP synthase, mitochondrial aldehyde dehydrogenase, mitochondrial superoxide dismutase, and heat shock proteins) were differentially expressed between LL, PM, and semitendinosus during postmortem aging. Future research validating the role of differentially abundant protein will improve the understanding on beef colour.

\section{Role of mitochondria versus mitochondrial enzymes in meat colour}

Immediately after animal harvest, apoptosis sets in and all organelles, including mitochondria, start to degenerate. Intact mitochondria with a dense matrix were observed immediately after slaughter (Cheah \& Cheah, 1971; Tang et al., 2005). However, as postmortem time increased, various morphological changes were noticed in the mitochondria. This suggests that mitochondrial enzymes can leak from the mitochondrial matrix into the sarcoplasm. Some of the oxidase enzymes can consume oxygen and lower oxygen partial pressure (Cornforth, 1994). This was evident in ground beef that was more colour unstable compared with intact steak that was more colour stable (Madhavi \& Carpenter, 1993). Grinding can release enzymes from the mitochondrial matrix and can decrease myoglobin redox states. However, limited knowledge is currently available on the role of mitochondrial matrix enzymes on meat discolouration. Furthermore, a compromised mitochondrial structure can limit MRA. For example, freeze-thawing of mitochondria decreased mitochondriamediated MRA by $41 \%$ in vitro (Tang et al., 2006). Freeze-thawing reduced colour stability (redness) of beef semimembranosus muscle by $44 \%$ on day 7 of storage in PVC packaging (Jeong et al., 2011).

\section{Oxygen consumption properties of game meat}

Although several studies have characterized game meat quality, limited knowledge is available on the wide diversity of game meat colour, and few details are available for mitochondrial and MRA properties. In general, game meat is darker in colour possibly, in part, due to greater pigment concentration. One might speculate that the muscle fibres would be more oxidative in nature due to greater physical activity. Conversely, studies have shown that game meat has more glycolytic muscles than oxidative fibres (Curry et al., 2012). A recent study characterized muscle-specific effects on colour stability of blesbok meat. The results indicated that the infraspinatus muscle was more colour stable than the longissimus thoracis et lumborum and biceps femoris (Neethling et al., 2016). Greater colour stability in the infraspinatus muscle was attributed to greater $\mathrm{pH}$ and MRA and lower OC on day 8 of storage compared with other muscles. However, in beef, longissimus is a colour-stable muscle compared with most other muscles. This suggests species-specific effects on discolouration. 


\section{Conclusions}

The functionality of mitochondria and a system for metmyoglobin reduction are highly interconnected with the redox states of myoglobin. The dynamic nature of postmortem muscle can stimulate a challenging study of the interactions between mitochondria and myoglobin. Use of high-throughput techniques such as proteomics and metabolomics should help elucidate the factors affecting mitochondrial function in postmortem muscle. Furthermore, the use of appropriate techniques to quantify tissue oxygen utilization and metmyoglobin reducing activity will also significantly enhance existing knowledge of the inter-relationships of these two key factors affecting meat colour. Understanding the factors involved in the mitochondrial function and myoglobin reduction will help meat processors and purveyors to formulate pre- and post-harvest strategies to maximize the economic benefits associated with improving the appearance of muscle foods.

\section{Acknowledgements}

This research was supported by the Agriculture and Food Research Institute Grant 2017-67018-26479 from the USDA National Institute of Food and Agriculture programme.

\section{Authors' Contributions} manuscript.

RR designed and drafted manuscript; MNN drafted the manuscript; $\mathrm{MCH}$ and SPS critically evaluated the

\section{Conflict of Interest Declaration}

The authors declare that they have no affiliations with any organization or entity with any financial or non-financial interest that could bias the subject matter and outcomes discussed in this manuscript.

\section{References}

Abraham, A., Dillwith, J.W., Mafi, G.G., VanOverbeke, D.L. \& Ramanathan, R., 2017. Metabolite profile differences between beef longissimus and psoas muscles during display. Meat Mus. Bio. 1, 18-26.

AMSA., 2012. Meat color measurement guidelines. Am. Meat Sci. Assoc., Champaign, IL.

Arihara, K., Cassens, R.G., Greaser, M.L., Luchansky, J.B. \& Mozdiak, P.E., 1995. Localization of metmyoglobinreducing enzyme (NADH-cytochrome b5 reductase) system components in bovine skeletal muscle. Meat Sci. 39, 205-213.

Ashmore, C.R., Parker, E. \& Doerr, L., 1972. Respiration of mitochondria isolated from dark cutting beef: Postmortem changes. J. Anim. Sci. 34, 46-48.

Baron, C.P. \& Andersen. H.J., 2002. Myoglobin-induced lipid oxidation. A review. J. Agr. Food Chem. 50, 3887-3897.

Bekhit, A.E.D. \& Faustman, C., 2005. Metmyoglobin reducing activity. Meat Sci. 71, 407-439.

Bendall, J.R., 1972. Consumption of oxygen by the muscles of beef animals and related species, and its effect on the colour of meat. I. Oxygen consumption in pre-rigor muscle. J. Sci. Food Agr. 23, 61-72.

Belskie, K.M., Ramanathan, R., Suman, S.P. \& Mancini, R.A., 2015a. Effects of muscle type and display time on beef mitochondria. Meat Sci. 101, 157-158.

Belskie, K.M., Van Buiten, C.B., Ramanathan, R. \& Mancini, R.A., 2015b. Reverse electron transport effects on NADH formation and metmyoglobin reduction. Meat Sci. 105, 89-92.

Bjelanovic, M., Egelandsdal, B., Phung, V.T., Langsrud, Ø., Sørheim, O., Hunt, M. \& Slinde, E., 2016. Mitochondrial electron transport generated by Krebs cycle substrates changed the myoglobin redox forms in ground beef packaged in aerobic and anaerobic atmosphere. Food Pack. Sh. Life. 8, 24-32.

Canto, A.C.V.C.S., Suman, S.P., Nair, M.N., Li, S., Rentfrow, G., Beach, C.M., Silva, T.J.P., Wheeler, T.L., Shackelford S.D., Grayson, A., McKeith, R.O. \& King, D.A., 2015. Differential abundance of sarcoplasmic proteome explains animal effect on beef Longissimus lumborum color stability. Meat Sci. 102, 90-98.

Cheah, K.S. \& Cheah, A.M., 1971. Post-mortem changes in structure and function of ox muscle mitochondria. 1. Electron microscopic and polarographic investigations. J. Bio. Biomemb. 2, 85-92.

Cornforth, D., 1994. Color - its basis and importance. In: Quality Attributes and their Measurement in Meat, Poultry and Fish Products. 1st ed. Pearson, A.M. \& Dutson, T.R. (eds). Blackie Academic \& Professional: Glasgow, United Kingdom, pp. 34-78.

Cornforth, D.P. \& Egbert, W.R., 1985. Effect of rotenone and pH on the color of pre-rigor muscle. J. Food Sci. 50, 34-35.

Djimsa, B.A., Abraham, A., Mafi, G.G., VanOverbeke, D. \& Ramanathan, R., 2017. Effects of metmyoglobin reducing activity and thermal stability of $\mathrm{NADH}$-dependent reductase and lactate dehydrogenase on premature browning in ground beef. J. Food Sci. 82, 304-313.

Elroy, N.N., Rogers, J., Mafi, G.G., VanOverbeke, D.L., Hartson, S.D. \& Ramanathan, R., 2015. Species-specific effects on non-enzymatic metmyoglobin reduction. Meat Sci. 105, 108-113.

English, A.R., Wills, K.M., Harsh, B., Mafi, G.G., VanOverbeke, D.L. \& Ramanathan, R., 2016a. Effects of extended aging on biochemical properties of dark cutting beef. J. Animal Sci. 94, 4040-4048.

English, A.R., Mafi, G.G., VanOverbeke, D.L. \& Ramanathan, R., 2016b. Effects of extended aging and modified atmospheric packaging on beef top loin steak color. J. Anim. Sci. 94, 1727-1737.

Estabrook, R.W., 1967. Mitochondrial respiratory control and the polarographic measurement of ADP: O ratios. Methods Enzymol. 10, 41-47. 
Faustman, C. \& Cassens, R.G., 1990. The biochemical basis for discoloration in fresh meat: a review. J. Mus. Food. 1, 217-243.

Giddings, G.G., 1977. The basis of color in muscle foods. Crit. Rev. Food Sci. Nutr. 9, 81-114.

George, P. \& Stratmann, C.J., 1952. The oxidation of myoglobin to metmyoglobin by oxygen. 1. Biochem. J. 51, $103-108$.

Hunt, M.C. \& Hedrick. H.B., 1977. Profile of fibre types and related properties of five bovine muscles. J. Food Sci. 42 , 513-517.

Jayasingh, P., Cornforth, D.P., Carpenter, C.E. \& Whittier, D., 2001. Evaluation of carbon monoxide (CO) treatment in modified atmosphere packaging or vacuum packaging to increase color stability of fresh beef. Meat Sci. 59, 317-324.

Jayasingh, P., Cornforth, D.P., Brennand, C.P., Carpenter, C.E. \& Whittier, D.R., 2002. Sensory evaluation of ground beef stored in high-oxygen modified atmosphere packaging. J. Food Sci. 67, 3493-3496.

Jeong, J., Kim, G., Yang, H. \& Joo, S., 2011. Effect of freeze-thaw cycles on physicochemical properties and color stability of beef semimembranosus muscle. Food Res. Int. 44, 3222-3228.

Joseph, P., Suman, S.P., Rentfrow, G., Li, S. \& Beach, C.M., 2012. Proteomics of muscle specific beef color stability. J. Agr. Food Chem. 60, 3196-3203.

Ke, Y., Mitacek, R.M., Abraham, A., Mafi, G.G., VanOverbeke, D.L., DeSilva, U. \& Ramanathan, R., 2017. Effects of muscle-specific oxidative stress on cytochrome $c$ release and oxidation-reduction potential properties. J. Ag. Food Chem. 65, 7749-7755.

Kim, Y.H., Hunt, M.C., Mancini, R.A., Seyfert, M., Loughin, T.M., Kropf, D.H. \& Smith, J.S., 2006. Mechanism for lactatecolor stabilization in injection-enhanced beef. J. Agric. Food Chem. 54, 7856-7862.

King, D.A., Shackelford, S.D., Kalchayanand, N. \& Wheeler, T.L., 2012. Sampling and aging effects on beef longissimus color stability measurements. J. Anim. Sci. 90, 3596-3605.

Klingenberg, M., 1968. The respiratory chain. In: Biological Oxidation. Singer, T.P. (ed). Wiley-Interscience, New York, 3.

Lanari, M.C. \& Cassens, R.G., 1991. Mitochondrial activity and beef muscle color stability. J. Food Sci. 56, $1476-1479$.

Lawrie, R.A., 1961. Systematic analytical differences between psoas major and longissimus dorsi muscles of cattle. Br. J. Nutr. 15, 453-456.

Ledward, D.A., 1985. Post-slaughter influences on the formation of metmyoglobin in beef muscles. Meat Sci. 15, 149-171.

Ma, D., Kim, Y.H.B., Cooper, B., Oh, J., Chun, H., Choe, J., Schoonmaker, J.P., Ajuwon, K. \& Min, B., 2017. Metabolomics profiling to determine the effect of postmortem aging on color and lipid oxidative stabilities of different bovine muscles. J. Agric. Food Chem. 65, 6708-671

Madhavi, D.L. \& Carpenter, C.E., 1993. Aging and processing affect color, metmyoglobin reductase and oxygen consumption of beef muscles. J. Food Sci. 58, 939-942.

Mancini, R.A. \& Hunt, M.C., 2005. Current research in meat color. Meat Sci. 71, 100121.

Mancini, R.A. \& Ramanathan, R., 2014. Effects of postmortem storage time on color and mitochondria in beef. Meat Sci. 98, 65-70.

Mancini, R.A., Kim, Y.H., Hunt, M.C. \& Lawrence, T.E., 2004. How does lactate enhancement improve beef color stability? In: Proceedings of the 50th International Congress of Meat Science and Technology; University of Helsinki Department of Food Technology: Helsinki, Finland. p. 41.

McKenna, D.R., Mies, P.D., Baird, B.E., Pfeiffer, K.D., Ellebracht, J.W. \& Savell, J.W., 2005. Biochemical and physical factors affecting discoloration characteristics of 19 bovine muscles. Meat Sci. 70, 665-682.

McKeith, R.O., King, D.A., Grayson, A.L., Shackelford, S.D., Gehring, K.B., Savell, J.W. \& Wheeler, T.L., 2016. Mitochondrial abundance and efficiency contribute to lean color of dark-cutting beef. Meat Sci. 116, 165-173.

Mohan, A., Hunt, M.C., Barstow, T.J., Houser, T.A. \& Muthukrishnan, S., 2010a. Effects of malate lactate, and pyruvate on myoglobin redox stability in homogenates of three bovine muscles. Meat Sci. 86, 304-310.

Mohan, A., Hunt, M.C., Barstow, T.J., Houser, T.A., Bopp, C. \& Hueber, D.M., 2010b. Effects of fibre orientation, myoglobin redox form, and postmortem storage on NIR tissue oximeter measurements of beef longissimus muscle. Meat Sci. 84, 7985.

Mohan, A., Hunt, M.C., Muthukrishnan, S., Barstow, T.J. \& Houser, T.A., 2010c. Myoglobin redox form stabilization by compartmentalized lactate and malate dehydrogenases. J. Agric. Food Chem. 58, 7021-7029.

Mohan, A., Hunt, M.C., Barstow, T.J., Houser, T.A. \& Hueber, D.M., 2010d. Near-infrared oximetry of three post-rigor skeletal muscles for following myoglobin redox forms. Food Chem. 123, 456-464.

Nair, M.N., Suman, S.P., Chatli, M.K., Li, S., Joseph, P., Beach, C.M. \& Rentfrow, G., 2016. Proteome basis for intramuscular variation in color stability of beef semimembranosus. Meat Sci. 113, 9-16.

Nair, M.N., Ramanathan, R., Rentfrow, G. \& Suman, S.P., 2017a. Intramuscular variations in mitochondrial functionality and sarcoplasmic proteome profile of bovine semimembranosus. S. Afr. J. Anim. Sci. 47, 635-639.

Nair, M.N., Li, S., Beach, C.M., Rentfrow, G. \& Suman, S.P., 2017b. Changes in the sarcoplasmic proteome of beef muscles with differential color stability during postmortem aging. Meat Mus. Bio. 2, 1-17.

Neethling, N.E., Suman, S.P., Sigge, G.O. \& Hoffman, L.C., 2016. Muscle-specific colour stability of blesbok (Damaliscus pygargus phillipsi) meat. Meat Sci. 119, 69-79.

O'Keeffe, M. \& Hood. D.E., 1982. Biochemical factors influencing metmyoglobin formation on beef from muscles of differing colour stability. Meat Sci. 7, 209-228.

Phung, V.T., Khatri, M., Liland, K.H., Slinde, E., Sørheim, O., Almøy, T., Saarem, K. \& Egelandsdal, B., 2013. Mitochondrial oxygen consumption in permeabilized fibres and its link to colour changes in bovine $M$. semimembranosus muscle. Meat Sci. 93, 128-137. 
Raines, C.R., Hunt, M.C. \& Unruh, J.A., 2010. Contributions of muscles of various color stabilities to the overall color stability of ground beef. J. Food Sci. 75 (1), C85-C89.

Ramanathan, R., Mancini, R.A. \& Konda. M.K.R., 2009. Effects of lactate on beef heart mitochondrial oxygen consumption and muscle darkening. J. Agri. Food Chem. 57, 1550-1555.

Ramanathan. R., Mancini, R.A., Naveena, B.M. \& Konda. M.K.R., 2010a. Effect of lactate-enhancement on reflectance and absorbance of beef longissimus steaks. Meat Sci. 84, 219-226.

Ramanathan, R., Mancini, R.A. \& Konda. M.K.R., 2010b. Effect of lactate-enhancement on myoglobin oxygenation of beef longissimus steaks overwrapped in PVC and stored at $4{ }^{\circ} \mathrm{C}$. J. Muscle Foods. 21, 669-684.

Ramanathan, R. \& Mancini. R.A., 2010c. Effects of pyruvate on mitochondria-mediated metmyoglobin reduction. Meat Sci. 86, 738-741.

Ramanathan. R., Mancini, R.A. \& Naveena, B.M., 2010d. Effects of lactate on mitochondria-mediated metmyoglobin reduction. J. Agri. Food Chem. 58, 5724-5729.

Ramanathan, R., Mancini, R.A. \& Dady. G., 2011. Effects of pyruvate, succinate, and lactate on beef longissimus color. Meat Sci. 88, 424-428.

Ramanathan, R., Mancini, R.A., Suman, S.P. \& Cantino. M.E., 2012. Effects of 4-hydroxy-2-nonenal on beef heart mitochondrial ultrastructure, oxygen consumption, and metmyoglobin reduction. Meat Sci. 90, 564-571.

Ramanathan, R., Mancini, R.A., Joseph, P. \& Suman, S.P., 2013. Bovine mitochondrial oxygen consumption effects on oxymyoglobin in the presence of lactate as a substrate for respiration. Meat Sci. 93, 893-897.

Ramanathan, R., Mancini, R.A., Suman, S.P. \& Cantino. M.E., 2014. Covalent binding of 4-hydroxy-2-nonenal to lactate dehydrogenase decreases $\mathrm{NADH}$ formation and metmyoglobin reducing activity. J. Agric. Food Chem. 62, 2112-2117.

Sammel, L.M., Hunt, M.C., Kropf, D.H., Hachmeister, K.A., Kastner, C.L. \& Johnson, D.E., 2002a. Influence of chemical characteristics of beef inside and outside semimembranosus on color traits. J Food Sci. 67, 1323-1330.

Sammel, L.M., Hunt, M.C., Kropf, D.H., Hachmeister, K.A. \& Johnson, D.E., 2002b. Comparison of assays for metmyoglobin reducing ability in beef inside and outside semimembranosus muscle. J. Food Sci. 67, 978-984.

Seyfert, M., Mancini, R.A., Hunt, M.C., Tang, J. \& Faustman, C., 2007. Influence of carbon monoxide in package atmospheres containing oxygen on colour, reducing activity, and oxygen consumption of five bovine muscles. Meat Sci. 75, 432-442.

Smith, A.L. 1967. Preparation, properties, and conditions for assay of mitochondria: Slaughterhouse material, smallscale. Methods Enzymol. 10, 81-86.

Suman, S.P. \& Joseph. P., 2013. Myoglobin chemistry and meat color. Annu. Rev. Food Sci. 4, 79-99.

Suman, S.P., Hunt, M.C., Nair, M.N. \& Rentfrow. G., 2014. Improving beef color stability: Practical strategies and underlying mechanisms. Meat Sci. 98, 490-504.

Tang, J., Faustman, C., Hoagland, T.A., Mancini, R.A., Seyfert, M. \& Hunt, M.C., 2005a. Postmortem oxygen consumption by mitochondria and its effects on myoglobin form and stability. J. Agri. Food Chem. 53, 1223-1230.

Tang, J., Faustman, C., Mancini, R.A., Seyfert, M. \& Hunt, M.C., 2005b. Mitochondrial reduction of metmyoglobin: Dependence on the electron transport chain. J. Agric. Food Chem. 53, 5449-5455.

Wittenberg, B.A. \& Wittenberg. J.B., 1989. Transport of oxygen in muscle. Annu. Rev. Physiol. 51, 857-878.

Wills, K.M., Mitacek, R.M., Mafi, G.G., VanOverbeke, D.L., Jaroni, D., Jadeja, R. \& Ramanathan, R., 2017. Improving the lean muscle color of dark-cutting beef by aging, antioxidant-enhancement, and modified atmospheric packaging. J. Anim. Sci. 95, 5378-5387. 